FEATURE ARTICLE

\title{
Promoting HIV and sexual safety behaviour in people with severe mental illness: A systematic review of behavioural interventions
}

\author{
Chloe Walsh, ${ }^{1}$ Edward McCann, ${ }^{2}$ Simon Gilbody ${ }^{3}$ and Elizabeth Hughes ${ }^{4}$ \\ ${ }^{1}$ Hull and East Yorkshire Hospitals NHS Trust, ${ }^{3}$ Health Sciences, University of York and Hull-York Medical School, \\ ${ }^{4}$ Health Sciences, University of York, York, UK; and ${ }^{2}$ School of Nursing and Midwifery, Trinity College, University of \\ Dublin, Dublin, Ireland
}

\begin{abstract}
People diagnosed with severe mental illness (SMI) are at greater risk of HIV than the general population. However, little attention has been given to how best to reduce sexual risk taking in this group. The aim of the review was to evaluate the effectiveness of behavioural interventions to promote sexual safety behaviour in people diagnosed with SMI. A comprehensive search of relevant databases was undertaken, and studies were included if they were randomized, controlled trials; behavioural intervention related to sexual behaviour; included adults diagnosed with SMI; and if a behavioural outcome was reported. The Cochrane Assessment of Bias Tool was used. Of the initial 515 papers identified, 11 trials were included for quality assessment and data extraction. The studies were heterogeneous in content and dose of intervention, as well as outcome measure and follow-up periods, and all had some risk of bias. Four of the studies demonstrated significant improvement in safer sexual behaviour at follow up, but this effect diminished over time. The effect sizes were extremely variable. There is emerging evidence to suggest that a behavioural intervention has the potential to reduce sexual risks in people diagnosed with SMI. However, further high-quality research is needed in this area.
\end{abstract}

KEY WORDS: health promotion, HIV, intervention, review, schizophrenia.

\section{INTRODUCTION}

There has been conventional wisdom among mental health-care professionals that people diagnosed with severe mental illness (SMI) are not sexually active, and it remains a subject that is rarely discussed with service users (Hughes \& Gray 2009; McCann 2010; Quinn et al. 2011). However, a review of studies of HIV risk behaviours (Carey et al. 1997) reported that $54-74 \%$ of people diagnosed with SMI had been sexually active in the pre-

Correspondence: Elizabeth Hughes, Health Sciences, University of York, Area 4, ARRC Building, York YO10 5DD, UK. Email: elizabeth.hughes@york.ac.uk

Chloe Walsh, MA, MBBS, MRCP.

Edward McCann, RN, MSc, PhD.

Simon Gilbody, DPhil, FRCPsych, FRSA.

Elizabeth Hughes, BSc, RN, DipHE, PhD.

Accepted January 2014. ceding year. In addition, some were engaging in high-risk behaviours, such as trading sex, having multiple partners, having partners known to be intravenous drug users, and inconsistent condom use. The nature of the link between serious mental illness and high-risk sexual behaviour is likely to be complex and influenced by unstable psychiatric symptoms (such as hypersexuality), comorbid drug and alcohol problems (Meade \& Sikkema 2007), and childhood and adulthood sexual abuse and exploitation (Meade et al. 2009; Wilson \& Widom 2009). Prevalence studies indicate that people diagnosed with SMI might be at higher risk of sexually-transmitted infections (STI). Campos et al. (2008) reviewed HIV prevalence studies and found consistent evidence that people diagnosed with SMI were disproportionately affected by HIV. Prevalence rates in the USA ranged from $1.7 \%$ to $5 \%$ compared with general population infection, which was approximately 
$0.3-0.4 \%$. The range of rates reflects the differences in the demographics of the samples; for example, higher rates in younger homeless samples. Comorbidity between HIV and a serious mental illness, such as schizophrenia, poses particular challenges for both users and services; in particular, engagement with services and treatment adherence, as well as the psychiatric and neurological consequences compounding a pre-existing mental health problem (Angelino \& Treisman 2008). There is currently no cure for HIV infection; therefore, preventative measures are key in reducing the illness burden of HIV and other sexually-transmitted diseases in this group. Research has shown that improving sexual health knowledge alone is insufficient to bring about behavioural change. Behavioural interventions that address knowledge, skills, confidence, motivation, and behaviour have been shown to be more effective (Shield et al. 2005). Mental health nurses are in a prime position to provide this type of intervention as part of overall holistic care (Quinn et al. 2013).

\section{Rationale}

The aim of this systematic review was to evaluate the quality and effectiveness of psychosocial interventions targeting sexual safety behaviour (e.g. increased condom use, reduced unprotected sex) for people diagnosed with SMI in order to inform evidence-based mental health practice.

\section{METHODS}

Studies were included in the review according to the following PICO (Participant, Intervention, Comparator and Outcome) criteria (http://www.prisma-statement .org).

\section{Participants}

Participants included adults of any age diagnosed with SMI, who were treated in any psychiatric setting (including those who were homeless, and those with a dual diagnosis of alcohol/substance misuse). Studies of patients who only had a non-psychotic illness (e.g. depression) were excluded. SMI is commonly used in the literature, but is often ill-defined. For the purpose of this review, we defined patients with serious mental illness pragmatically, as those with a chronic, relapsing psychiatric disorder that requires treatment in secondary or specialist mental health setting. Therefore, we included those studies where the participants had a diagnosed psychiatric illness, such as schizophrenia, schizoaffective disorder, bipolar disorder, or major depression, and who were in receipt of secondary mental health care. We excluded any study where participants were in primary care or other nonpsychiatric health-care settings.

\section{Interventions}

We were interested in any type of non-pharmacological intervention aimed at promoting sexual safety-taking behaviours delivered in any psychiatric setting. The interventions of interest could include (but were not limited to) multisession psychosocial interventions, providing skills training in communication and assertiveness, motivational interviewing, and/or cognitive behavioural therapy in an attempt to reduce sexual risk taking behaviour; and standard education interventions aimed at improving knowledge to reduce sexual risk taking or minimal interventions, for example, the provision of written information about STI and risk-taking behaviour.

\section{Comparator}

Any comparison intervention or treatment as usual was used.

\section{Outcome}

Any outcome relating to sexual behaviour change was used. This could be measured objectively; for example, reduced incidence of STI; or subjectively, for example, self-reporting of measures, such as abstinence, reduced number of sexual partners, increased attendance at sexual health clinics, episodes of unprotected anal, oral, or vaginal sex, increased use of condoms, or reduced incidence of intercourse while intoxicated.

\section{Study design}

All relevant randomized, controlled trials (with or without blinding) were included. There was no restriction on publication date; no non-English language studies were identified. Studies to be included had to report findings for a control or comparison group; however, they were not excluded on the basis of the nature of the control.

\section{Search methods for identification of studies}

A systematic search of the following databases was undertaken from inception to May 2013: Cochrane, MEDLINE, EMBASE, PsychINFO, CINAHL, and BNI. No limits were placed on the search. The search terms used centred on three themes:

- Safe sex OR unsafe sex OR sexual risk taking OR risk behaviour OR STI OR HIV OR chlamydia OR gonorrhoea OR hepatitis OR syphilis OR acquired immunodeficiency syndrome OR condoms OR barrier contraception AND 
- Behavioural therapy OR cognitive therapy OR psychosocial intervention OR skills training OR sex education OR motivational interviewing AND

- Severe/serious/chronic mental illness OR schizophrenia OR bipolar OR mania OR psychosis OR schizoaffective.

A total of 515 studies were identified on database searching. Duplicates were removed using EndNote bibliographic software. The remaining abstracts were screened against the inclusion criteria set out above by one researcher, and those deemed irrelevant were excluded at this stage. Full texts for those identified as potentially relevant were then obtained and screened by two reviewers (EH and EM) independently, before meeting to agree which studies should be included in the review. Reasons for exclusion at this stage included lack of control group, participants without SMI, and the outcome measure being one of knowledge rather than behaviour. Disagreements were handled by discussion between the reviewers and with a third reviewer, as needed, resulting in a final total of included studies (see Fig. 1). The authors of relevant papers were contacted for information about ongoing research relevant to the review or unpublished data in the area to minimize the risk of selective reporting. Citation searching for studies meeting the inclusion criteria was conducted, and the reference list of each included study was visually scanned to look for further relevant papers. A data extraction tool was developed and piloted on two papers by two separate reviewers. For each included study, two independent researchers undertook data extraction using a standard form, and then met to discuss and agree on the content.

\section{Data collection and analysis}

Data were extracted under the following headings: setting (type of service, geographical location), participant characteristics (diagnosis, gender mix, sample size), attrition rate (percentage lost to follow up), type of intervention (group, type of intervention), dose (length and frequency), control or comparator arm, and sexual behaviour outcomes for groups at follow up. Results for all behavioural outcomes from included studies are reported. Mean values are reported where possible, and $P$-values are given for all significant behavioural outcomes (Table 1).

\section{Assessment of risk of bias in included studies}

The methodological quality of the trials was assessed using the Cochrane Risk of Bias Assessment Tool (Higgins 2008). All studies were included in the review,

FIG. 1: Flowchart for retrieval and inclusion of papers.

Included studies $n=11$
Excluded $n=36$

Unable to obtain $=4$

Not relevant design $=17$

Report of trial considered elsewhere $=6$

No behavioural outcome $=5$

Patients with no severe mental illness $=3$ No intervention $=1$ 


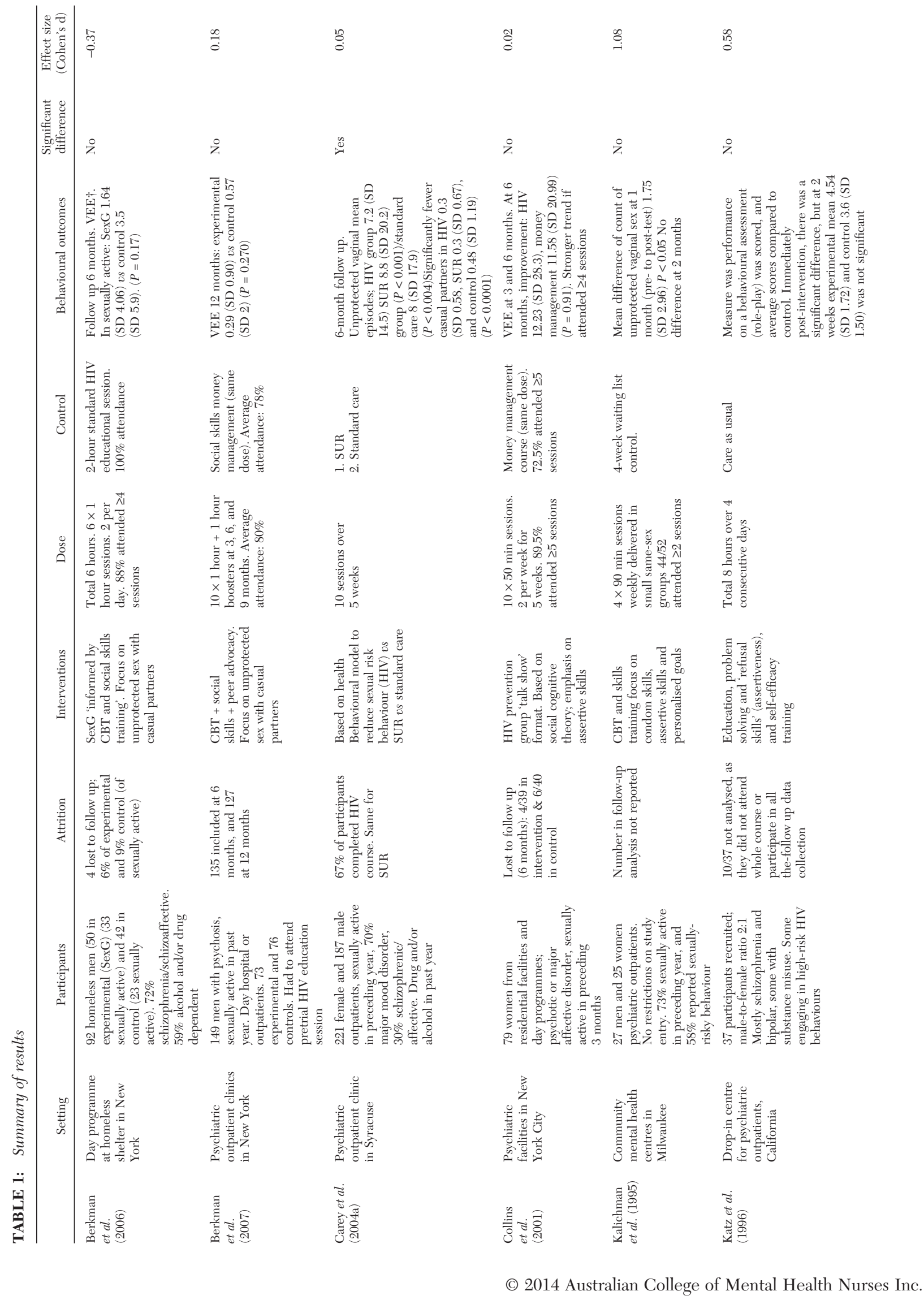




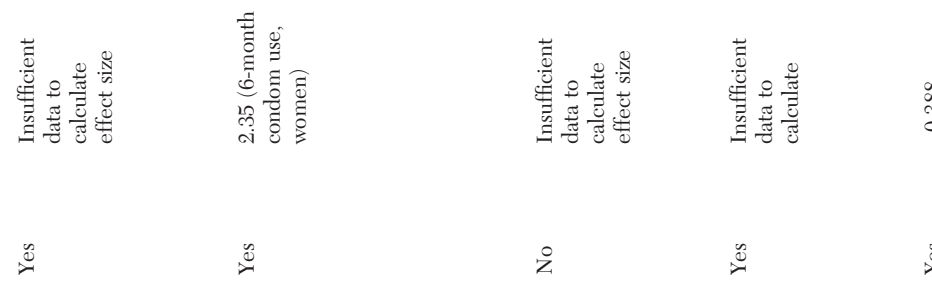
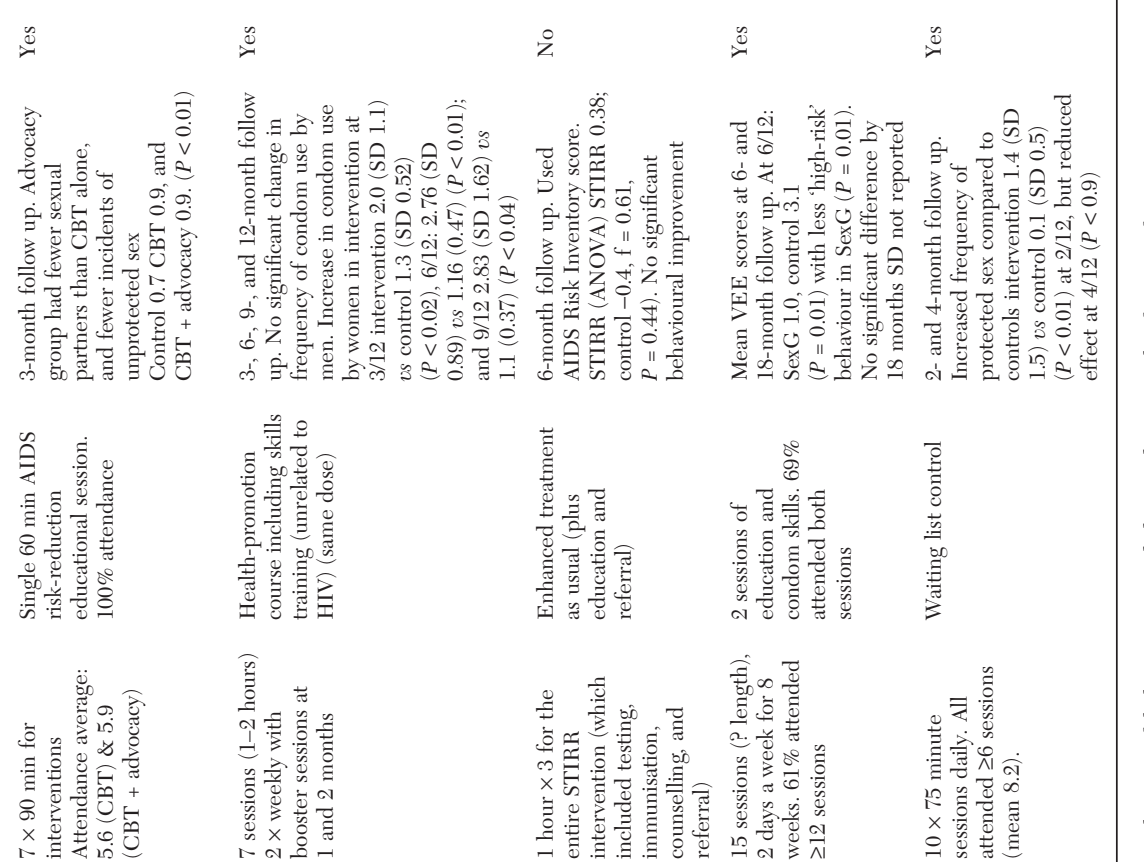

MUEn
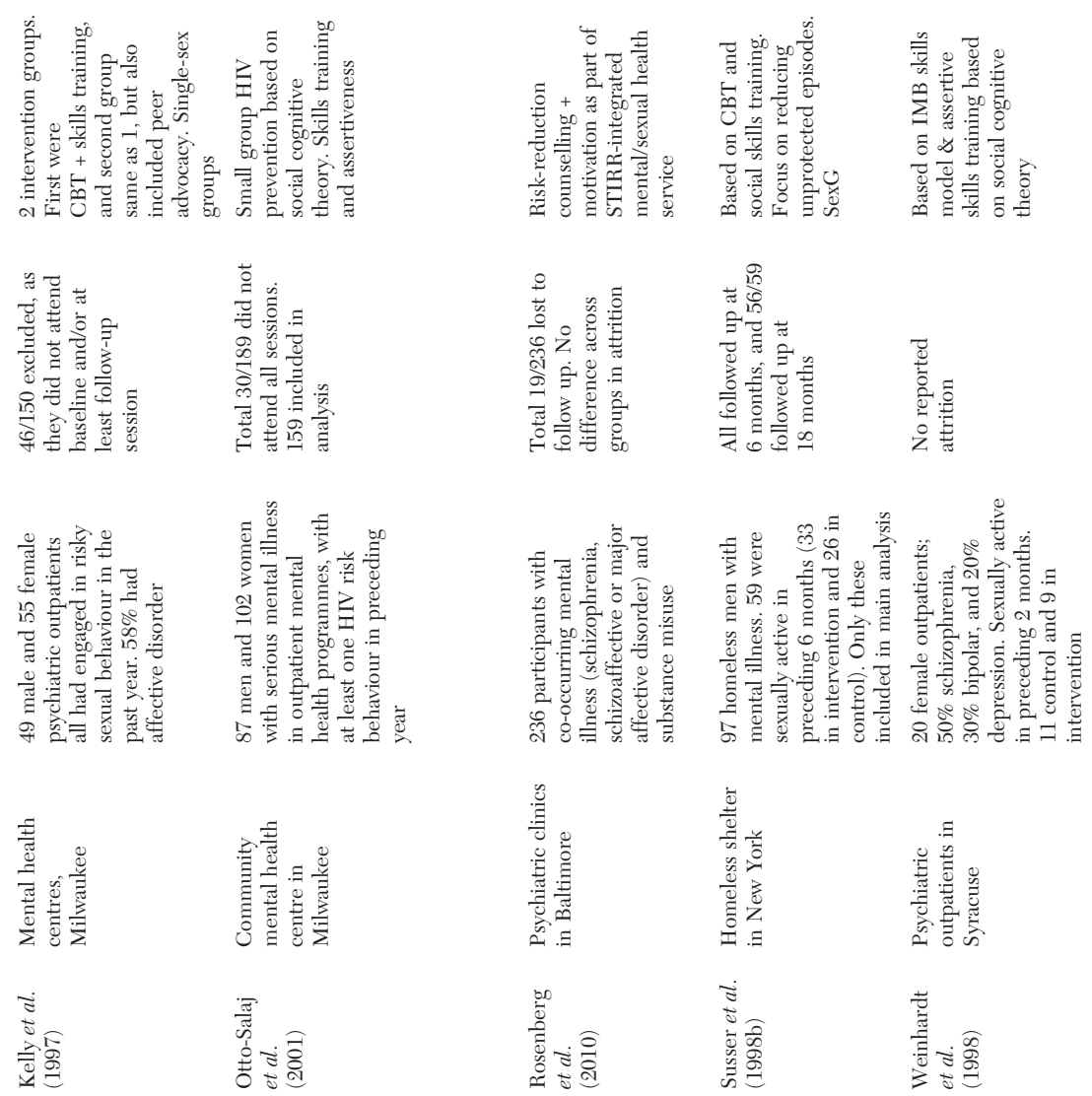

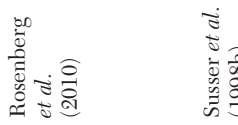
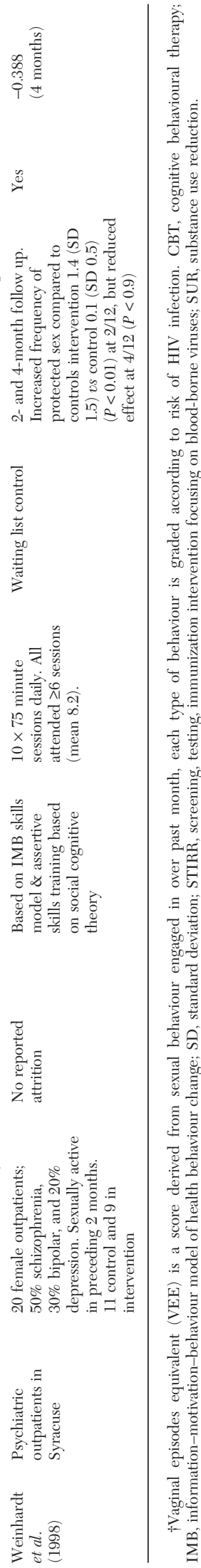
regardless of quality. However, in the discussion of the results, more weight was given to the studies of stronger design.

\section{RESULTS}

Initial searching yielded 515 references. After removal of duplicates and screening of titles and abstracts, full papers for 45 studies were obtained. Of these, a total of 11 studies were identified, which satisfied our inclusion criteria for data extraction and assessment of risk of bias. These were all randomized, controlled trials, and all undertaken in the USA between 1995 and 2013. Due to variations in types of outcome measures, timing of follow-up data collection, and the heterogeneous nature of both intervention and participants (gender, diagnostic mix, and type of service setting) a meta-analysis could not be performed.

\section{Risk of bias}

A summary of the risk of bias assessment is shown in Figure 2. All studies were judged by the Cochrane Risk of Bias tool to be of high risk of bias in at least one domain, as all used self-reported measures of behaviour. However this seems unavoidable, given the nature of the outcome being reported. Many studies were rated 'amber', as there was insufficient detail in the reporting of the methods section regarding areas of potential bias, such as concealment and allocation. Furthermore, several studies had high attrition rates (median drop-out rates $=8 \%$, range $=$ 0-27\%), and only three studies used an intention-to-treat analysis. Furthermore, some of the studies had small samples and might have been underpowered to detect significant differences between groups.

\section{Outcome measures}

The type of outcome measures used for gathering data for sexual risk behaviour was variable across studies. All relied on self-report with no objective indicator of sexual risk behaviour (e.g. incidence of STI); however, Sohler et al. (2000) found that self-report is reasonably reliable in homeless men diagnosed with SMI. The most commonly reported outcome was the Vaginal Episodes Equivalent (VEE) sexual risk index (Susser et al. 1998a), which calculates a risk score based on the type of sexual risk behaviour that a person had engaged in over a specified time period, with unprotected anal sex as the most risky for HIV infection. This has been validated as a reliable index of sexual risk (Susser et al. 1998a). Other studies in this review used simple counting of incidents of unprotected sex and the use of condoms, and calculated a total score, which was used for analysis (see Table 1).

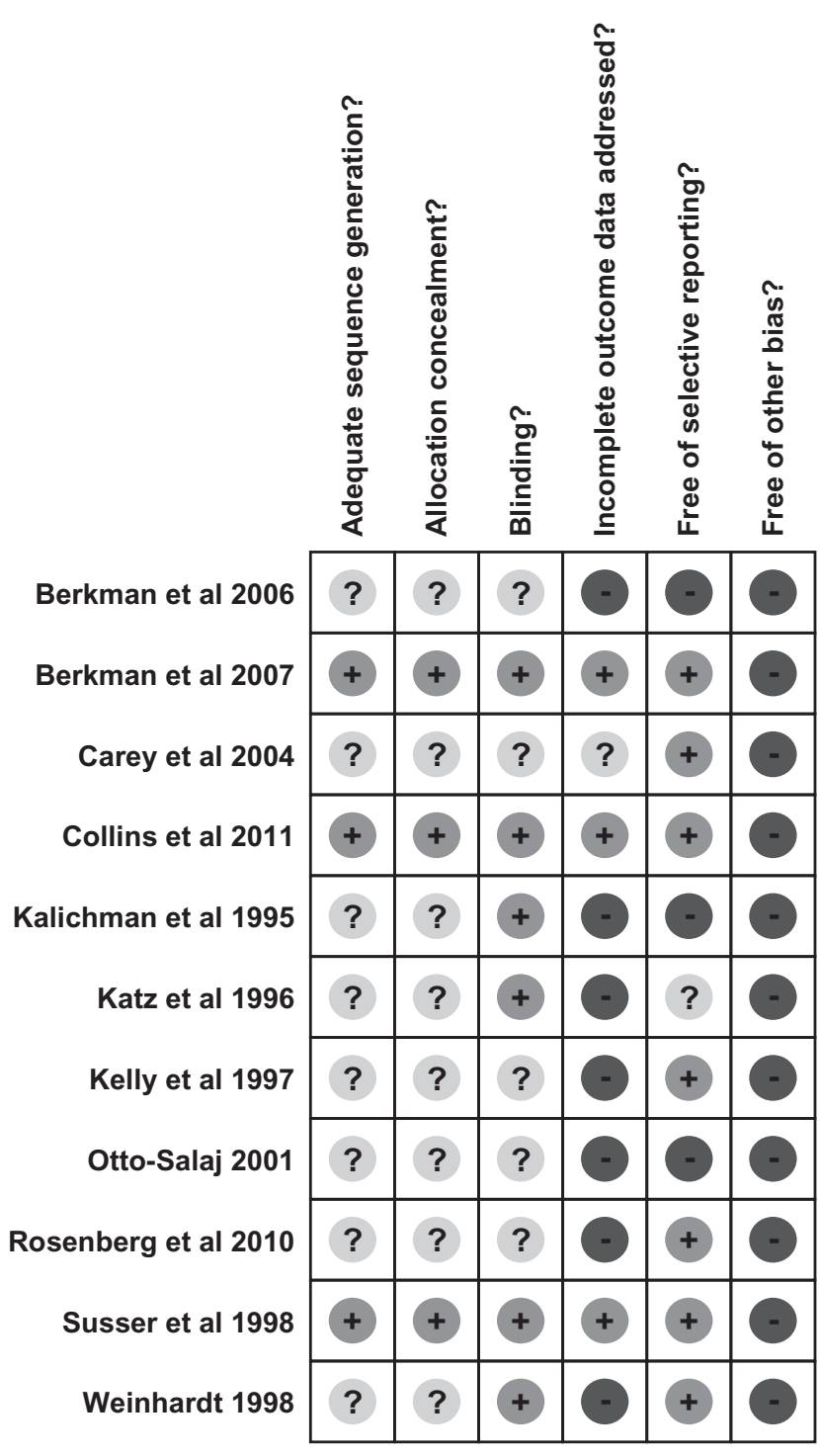

FIG. 2: Assessment of bias summary.

\section{Interventions}

Almost all the trials (10/11) were composed of group psychosocial interventions. The delivery of the group interventions varied in intensity, frequency, and content, but did have unifying characteristics: (i) knowledge and educational interventions to increase awareness of sexual health risks, HIV infection, and how to prevent infection; (ii) social skills development focusing on assertiveness and safer sex negotiation; (iii) behavioural skills, such as how to use a condom; and (iv) active participation, such as role-play, working through scenarios, and watching videos.

In addition, the dose of the interventions varied widely, ranging from three brief sessions (Rosenberg et al. 2010) through to 15 sessions (Susser et al. 1998b). 


\section{Effectiveness of interventions}

Seven of the 11 studies demonstrated statisticallysignificant improvements in sexual risk behaviour outcomes immediately upon completion of the intervention. (Carey et al. 2004a; Kalichman et al. 1995; Katz et al. 1996; Susser et al. 1998b; Weinhardt et al. 1998) (see Table 1). These outcomes included increased use of condoms, less sexual partners, and less unprotected sexual encounters. However, medium- and long-term differences were harder to detect, with only four studies demonstrating significant changes to sexual behaviour measured beyond 1-month post-intervention (Carey et al. 2004a; Gordon et al. 1999; Otto-Salaj et al. 2001; Susser et al. 1998b), and behaviour change was not maintained over the longer term (beyond 6 months) in any study.

\section{Intervention themes}

\section{Motivational enhancement}

Two studies (Carey et al. 2004a; Weinhardt et al. 1997) incorporated motivational-enhancement approaches within their group intervention, and both showed a significant improvement in sexual behaviour. Motivational interviewing (MI) (Miller \& Rollnick 2009) addresses readiness to change by looking at importance (seeing change as important right now, and that benefits outweigh costs), and also in terms of confidence (self-efficacy). One trial (Weinhardt et al. 1997) incorporated motivational approaches, as well as assertiveness, and will be discussed in the next section. The same research team subsequently developed a larger trial, which is described next (Carey et al. 2004a). They adopted the principles of MI, such as empathy and unconditional positive regard, as well as specific exercises to help develop motivation to adopt safer sexual practices, such as the pros and cons of condom use. The randomized trial had three arms: HIVfocused intervention; substance-use intervention, and standard care. They recruited participants with serious mental illness (which they defined as major mood or thought disorder) from mental health-care services in Syracuse (USA), who screened positive for recent sexual risk behaviour and substance use. The HIV-focused and substance-use interventions both consisted of twice-weekly, single gender groups over 5 weeks. The participants completed baseline measures of knowledge, decisional balance scale (motivation), behavioural intentions, and behavioural skills (via video role play assessment). Participants were followed up 1-2 weeks post-intervention, and then at 3 months and 6 months. Carey et al. (2004a) found there were significantly less mean episodes of unprotected vaginal sex in the HIV group $(P<0.001)$ compared to substance use or standard care $(P<0.004)$, and significantly fewer casual partners $(P<0.0001)$ in the HIV group over time. However, despite these behavioural changes, the motivation scores were not significantly different between groups. Therefore, behaviour change had occurred without a noticeable shift in motivation. Out of a total sample of people who fulfilled the inclusion criteria, $41 \%$ agreed to participate in the programme. This left $59 \%$ who were identified as engaging in high-risk sexual behaviour and using substances, but who declined to participate.

\section{Promoting assertiveness}

Three studies (Otto-Salaj et al. 2001; Weinhardt et al. 1997) focused on developing assertiveness in sexual relationships, as it is a common social deficit in people (particularly women diagnosed with SMI) (Collins et al. 2001; Weinhardt et al. 1998) also focused on assertiveness in the women-only trial 'Ourselves, Our Bodies', but failed to find a significant reduction in sexual behaviour, as measured by the Vaginal Episodes Equivalent (VEE) index (Susser et al. 1998a). In a pilot trial, Weinhardt et al. $(1997,1998)$ delivered an intervention based on the development of assertiveness and the art of negotiating safer sexual practices. They recruited 20 women diagnosed with SMI from a psychiatric outpatient service, who were then randomized to receive either the HIV intervention or waiting list control. The intervention was a 10-session programme, comprising of three sessions of education and building motivation, and then seven sessions focusing on assertiveness skills development and application in real-life situations. They reported that there was a significant increase in (self-reported) protected sex (i.e. use of condoms) after 2 months $(P>0.01)$, but this was diminished by 4 months $(P=0.9)$. Otto-Salaj et al. (2001) recruited people with a diagnosis of serious mental illness with at least one HIV infection risk factor in the previous year to be part of either a HIV intervention group or a health-promotion group (without sexual health content). The intervention and control were matched on time and attention. There were seven sessions (1-2 hours) delivered twice weekly, with booster sessions added at 1 and 2 months' post-intervention. There were no significant differences in sexual behaviour between groups among the male participants, but the female participants in the HIV group showed significant condom use frequency at 3 months' $(P=0.02), 6$ months' $(P=0.01)$, and 9 months' $(P=0.04)$ follow up, but not at 12 months' follow up.

Peer advocacy

Two studies added peer advocacy (Berkman et al. 2007; Kelly et al. 1997) to their respective intervention 
programmes. Their theoretical justification was that positive sexual health messages are more internalized and acted upon if the person becomes an agent for promoting healthier choices for others. Kelly et al. (1997) sought to investigate the effects of adding peer advocacy to a HIV group intervention, which included the use of cognitive behavioural therapy (CBT) techniques and social skills training. The analysis demonstrated a significant difference in favour of the group with the additional peer advocacy component at 3 months' post-intervention. Berkman et al. (2007) added peer advocacy to a 10-session HIVprevention group programme for outpatients with serious mental illness (included regardless of whether sexually active or not). This was adapted from a previouslysuccessful programme delivered to homeless males with serious mental illness (Susser et al. 1998b). The outcome measure was the VEE score. The comparison group was a time- and attention-matched money management and social skills training intervention. The intervention also included booster sessions at 3, 6, and 9 months' postintervention (Susser et al. 1998b). However, Berkman et al.'s (2007) study failed to find significant differences in sexual risk behaviours, as measured by the VEE score at the 6- and 12-month time points, and between those who had HIV prevention and peer advocacy compared with the comparison. When the subgroup of sexually-active participants was analysed, there was a strong effect size in this group for the HIV-reduction intervention, but this failed to reach significance at the 6- and 12-month time points.

\section{Individual intervention}

Most of the studies identified were group interventions. Only one study out of 11 evaluated an individuallydelivered intervention. It was debated whether this should be included in the final set of papers, as promoting safer sexual behaviour was only a small component of the whole intervention. However, as it was the only individual intervention, the decision was made by the review team to include. Rosenberg et al. (2010) conducted a trial of a new service, which comprised screening, testing, and immunization focused on blood-borne viruses (known as STIRR). This was targeted specifically at people with SMI who also had comorbid substance misuse problems because they are considered a high-risk group for bloodborne virus infection (Rosenberg et al. 2001). The STIRR intervention was delivered in addition to routine mental health care. It was composed of three 1:1 sessions over 6 months, and this mostly focused on clinical interventions (e.g. testing for blood-borne viruses and offering hepatitis B immunization), but also included discussions aimed at increasing awareness of risks and promoting behaviour change in relation to blood-borne viruses. Despite showing high recruitment and retention, as well as uptake of testing and vaccination in STIRR (compared with treatment as usual), there was no significant difference in sexual behaviour outcomes. This might be because of the brevity of the intervention.

\section{DISCUSSION}

The aim of the systematic review was to establish the effectiveness of psychosocial interventions focused on the sexual behaviour of people with SMI in increasing sexual safety behaviours. The search revealed limited published work in this area. Eleven randomized, controlled trials of weak-to-moderate quality were identified, which fulfilled the inclusion criteria. All had been conducted in the USA between 1995 and 2013, and generated by three research centres. The trials were heterogeneous, both in participant characteristics and type of intervention, including content, dose, setting outcome measures, and follow-up time points; therefore, a meta-analysis was not possible. Given that the studies are potentially biased and heterogeneous, the findings from the studies must be viewed with some caution. Overall, seven of 11 studies showed some statistically-significant impact on sexual behaviour outcomes immediately after completion, compared with control or comparator, and only four trials showed significant differences at follow up. Where follow-up periods were greater than 6 months' post-test, the significance differences disappeared. In addition, effect sizes were calculated using statistics reported in the papers (where possible), and these were extremely variable (Table 1 ).

The results should be interpreted in the context of common methodological shortcomings, namely small sample sizes, sample attrition, and possible bias from allocation and lack of blinding. However, it is promising that even where statistically-significant results were not found, there was an overall trend in the direction of the reduction of unsafe sexual behaviour and an increase in condom use. This is clinically important, as any preventative behaviour reduces the risk of infection. Trials in this review also demonstrated that people with SMI are able to willingly engage in these types of interventions, and make some changes to their behaviour as a result. However, not all those eligible for the trials agreed to participate. For example, in one study (Carey et al. 2004a), only $41 \%$ of those who screened positive for the trial agreed to participate. This then poses the question of how to address sexual behaviour for those who are perhaps less motivated to volunteer for this type of 
intervention. Reassuringly, those trials, which looked at the effect of the intervention on people who were not sexually active at baseline, showed that attending the intervention did not increase their sexual risk-taking behaviour (Susser et al. 1998b). Some of the studies targeted people with at least one recent sexual risk factor, and this seemed to demonstrate the most change in sexual behaviour at follow up. It was interesting to note that two demographic characteristics seemed to influence outcomes in some of the studies: gender and type of mental illness. In two trials (Katz et al. 1996; Weinhardt et al. 1997), with both male and female participants, the participants' data could be analysed by gender. The results of those trials found that that women were more likely to reduce sexual risk taking following participation in the interventions compared to men. One possibility is that the group format suited women service users better, and there is a possibility that men might benefit from individually-delivered sessions. Gender differences in response to psychosocial interventions should be further explored. Carey et al. (2004b) compared outcomes from those with a psychotic (schizophrenia, schizoaffective) disorders to those with a non-psychotic disorder (mood disorders), and found that those with a mood disorder were more likely to benefit from a specific group intervention than those who had psychotic disorders. The intervention needs to be adapted to accommodate some of the specific difficulties and barriers that people with SMI might face in considering health behaviour change, which include the cognitive and affective effects of antipsychotic medication, negative symptoms, low self-esteem and stigma, and cognitive deficits. These deficits might impede the ability to digest new information, to plan ahead, reflect on behaviour and consequences, and translate learning from role-play into real-life situations (Bellack \& DiClemente 1999). Other psychosocial interventions using MI in people with SMI have shown equivocal effects in reducing substance misuse (Barrowclough et al. 2010) and adherence to medication (Gray et al. 2006). MI seems to be most effective when there is a more immediate negative consequence to a specific behaviour. For example, in the MIDAS trial (Barrowclough et al. 2010), people with SMI who drank alcohol responded better to MI approaches. Bingeing on alcohol results in immediate unpleasant consequences, such as a hangover, whereas the negative effects of other drugs, such as cannabis, are more subtle and long term (e.g. risk of cancer, psychotic breakdown). Therefore, individuals with sexual risk behaviour might be less responsive to motivational approaches, as the negative consequences of unprotected sex (the realization of infection with an STI or HIV) might take weeks, months, or even years after the event.

Follow-up times were relatively short in the main, with few trials assessing impact beyond 6 months. One study with homeless males (Susser et al. 1998b) had the longest follow up at 18 months post-intervention, but failed to find any significant differences between groups beyond 6 months. How to maintain benefits over the long term (i.e. longer than 12 months) needs further evaluation. One possibility might be to develop a greater awareness of sexual risks and prevention within the mental health workforce, so that these questions become part of routine screening, assessment, and if necessary, targeted within the overall care plan. Some studies included booster sessions (Berkman et al. 2007; Kelly et al. 1997), but neither of these studies provided significant results. However, the effect of booster sessions should be investigated further in future trials.

\section{LIMITATIONS OF THE REVIEW}

There are some limitations to this review. Only published articles were included, and these were English-language only. This has the potential to exclude studies published in non-English-speaking countries. In addition, we did not search grey literature and unpublished literature, so might miss work because of this. We did contact as many authors as possible to check if there were any ongoing studies. In addition, the search was originally undertaken in 2011, but was repeated in May 2013 to check for new publications, but none were found.

\section{IMPLICATIONS FOR FUTURE NURSING RESEARCH AND PRACTICE}

Despite the mixed findings of the review, it is clear that people diagnosed with SMI are willing and able to engage with interventions that focus on sensitive subjects, such as sexual behaviour. Therefore, mental health nurses should recognize the potential opportunities that exist to develop their roles within sexual health promotion and recovery. Further research should address the issues raised in this review in terms of: (i) high-quality trial methodology to reduce the risk of bias; (ii) effective identification and recruitment of 'at-risk' people with SMI, so that the right people receive the 'right' intervention; (iii) development of the content of the intervention to maximize its benefits, particularly attending to the specific needs of people with psychotic disorders and addressing the gender differences that have been identified in the reviewed studies; (iv) exploring the dose and intensity of interventions to use 
the least intense intervention that optimizes behaviour change in the most cost-effective way; and (v) exploring long-term maintenance of safer sexual behaviours, including the addition and effectiveness of booster sessions, and other methods of promoting safer sex (e.g. poster campaigns, peer advocacy).

In conclusion, there is limited evidence that psychosocial interventions for people with SMI have a significant impact on improving sexual risk behaviour in the long term. Some trials have shown significant short-term effects on behaviour; however, the trials identified were of poor-to-moderate quality. In addition, all the outcomes were based on self-reported behaviour, and none used objective measures of sexual behaviour (e.g. infection with STI). However, given the severity of the consequences of someone with SMI being infected with HIV or other blood-borne viruses, there is a need to conduct more research that can feasibly be implemented in routine mental health-care settings.

\section{ACKNOWLEDGEMENTS}

This review was undertaken by Dr Chloe Walsh as part of her academic placement. We would also like to acknowledge the time that the co-authors have given to this review.

\section{REFERENCES}

Angelino, A. F. \& Treisman, G. J. (2008). Issues in co-morbid severe mental illnesses in HIV infected individuals. International Review of Psychiatry, 20, 95-101.

Barrowclough, C., Haddock, G., Wykes, T. et al. (2010). Integrated motivational interviewing and cognitive behavioural therapy for people with psychosis and comorbid substance misuse: Randomised controlled trial. BMJ (Clinical Research Ed.), 341, c6325.

Bellack, A. S. \& Diclemente, C. C. (1999). Treating substance abuse among patients with schizophrenia. Psychiatric Services, 50, 75-80.

Berkman, A., Cerwonka, E., Sohler, N. \& Susser, E. (2006). A randomized trial of a brief HIV risk reduction intervention for men with severe mental illness. Psychiatric Services, 57, 407-409.

Berkman, A., Pilowsky, D., Zybert, P. et al. (2007). HIV prevention with severely mentally ill men: A randomised controlled trial. AIDS Care, 19, 579-588.

Campos, L. N., Guimaraes, M. D., Carmo, R. A. et al. (2008). HIV, syphilis, and hepatitis B and C prevalence among patients with mental illness: A review of the literature. Cadernos De Saude Publica, 24 (Suppl. 4), s607-s620.

Carey, M. P., Carey, K. B. \& Kalichman, S. C. (1997). Risk for human immunodeficiency virus (HIV) infection among persons with severe mental illnesses. Clinical Psychology Review, 17, 271-291.

Carey, M. P., Carey, K. B., Maisto, S. A., Gordon, C. M., Schroder, K. E. \& Vanable, P. A. (2004a). Reducing HIV-risk behavior among adults receiving outpatient psychiatric treatment: Results from a randomized controlled trial. Journal of Consulting \& Clinical Psychology, 72, 252-268.

Carey, M. P., Carey, K. B., Maisto, S. A., Gordon, C. M., Schroder, K. E. E. \& Vanable, P. A. (2004b). Reducing HIV-risk behavior among adults receiving outpatient psychiatric treatment: Results from a randomized controlled trial. Journal of Consulting and Clinical Psychology, 72, 252-268.

Collins, P. Y., Geller, P. A., Miller, S., Toro, P. \& Susser, E. S. (2001). Ourselves, our bodies, our realities: An HIV prevention intervention for women with severe mental illness. Journal of Urban Health, 78, 162-175.

Gordon, C. M., Carey, M. P., Carey, K. B., Maisto, S. A. \& Weinhardt, L. S. (1999). Understanding HIV-related risk among persons with a severe and persistent mental illness: Insights from qualitative inquiry. Journal of Nervous \& Mental Disease, 187, 208-216.

Gray, R., Leese, M., Bindman, J. et al. (2006). Adherence therapy for people with schizophrenia. European multicentre randomised controlled trial. British Journal of Psychiatry, 189, 508-514.

Higgins, J. P. A. A. D. G. (2008). Assessing risk of bias in included studies. In: J. P. A. G. S. Higgins (Ed.). Cochrane Handbook for Systematic Reviews of Interventions: Cochrane Book Series. Chichester, UK: John Wiley \& Sons, Ltd.

Hughes, E. \& Gray, R. (2009). HIV prevention for people with serious mental illness: A survey of mental health workers' attitudes, knowledge and practice. Journal of Clinical Nursing, 18, 591-600.

Kalichman, S. C., Sikkema, K. J., Kelly, J. A. \& Bulto, M. (1995). Use of a brief behavioral skills intervention to prevent HIV infection among chronic mentally ill adults. Psychiatric Services, 46, 275-280.

Katz, R. C., Westerman, C., Beauchamp, K. \& Clay, C. (1996). Effects of AIDS counseling and risk reduction training on the chronic mentally ill. AIDS Education and Prevention, 8, 457-463.

Kelly, J. A., McAuliffe, T. L., Sikkema, K. J. et al. (1997). Reduction in risk behavior among adults with severe mental illness who learned to advocate for HIV prevention. Psychiatric Services, 48, 1283-1288.

McCann, E. (2010). The sexual and relationship needs of people who experience psychosis: Quantitative findings of a UK study. Journal of Psychiatric \& Mental Health Nursing, 17, 295-303.

Meade, C. S. \& Sikkema, K. J. (2007). Psychiatric and psychosocial correlates of sexual risk behavior among adults with severe mental illness. Community Mental Health Journal, 43, 153-169.

Meade, C. S., Kershaw, T. S., Hansen, N. B. \& Sikkema, K. J. (2009). Long-term correlates of childhood abuse among 
adults with severe mental illness: Adult victimization, substance abuse, and HIV sexual risk behavior. AIDS and Behavior, 13, 207-216.

Miller, W. R. \& Rollnick, S. (2009). Ten things that motivational interviewing is not. Behavioural \& Cognitive Psychotherapy, 37, 129-140.

Otto-Salaj, L. L., Kelly, J. A., Stevenson, L. Y., Hoffmann, R. \& Kalichman, S. C. (2001). Outcomes of a randomized smallgroup HIV prevention intervention trial for people with serious mental illness. Community Mental Health Journal 37, 123-144

Quinn, C., Happell, B. \& Browne, G. (2011). Talking or avoiding? Mental health nurses' views about discussing sexual health with consumers. International Journal of Mental Health Nursing, 20, 21-28.

Quinn, C., Happell, B. \& Welch, A. (2013). Talking about sex as part of our role: Making and sustaining practice change. International Journal of Mental Health Nursing, 22, 231240.

Rosenberg, S. D., Goodman, L. A., Osher, F. C. et al. (2001). Prevalence of HIV, hepatitis B, and hepatitis C in people with severe mental illness. American Journal of Public Health, 91, 31-37.

Rosenberg, S. D., Goldberg, R. W., Dixon, L. B. et al. (2010). Assessing the STIRR model of best practices for blood-borne infections of clients with severe mental illness.

Shield, H., Fairbrother, G. \& Obmann, H. (2005). Sexual health knowledge and risk behaviour in young people with first episode psychosis. International Journal of Mental Health Nursing, 14, 149-154.

Sohler, N., Colson, P. W., Meyer-Bahlburg, H. F. \& Susser, E. (2000). Reliability of self-reports about sexual risk behavior for HIV among homeless men with severe mental illness. Psychiatric Services (Washington, DC), 51, 814-816.

Susser, E., Desvarieux, M. \& Wittkowski, K. M. (1998a). Reporting sexual risk behavior for HIV: A practical risk index and a method for improving risk indices. American Journal of Public Health, 88, 671-674.

Susser, E., Valencia, E., Berkman, A. et al. (1998b). Human immunodeficiency virus sexual risk reduction in homeless men with mental illness. Archives of General Psychiatry, 55, 266-272.

Weinhardt, L. S., Carey, M. P. \& Carey, K. B. (1997). HIV risk reduction for the seriously mentally ill: Pilot investigation and call for research. Journal of Behavior Therapy and Experimental Psychiatry, 28, 87-95.

Weinhardt, L. S., Carey, M. P., Carey, K. B. \& Verdecias, R. (1998). Increasing assertiveness skills to reduce HIV risk among women living with a severe and persistent mental illness. Journal of Consulting and Clinical Psychology, 66, 680-684.

Wilson, H. W. \& Widom, C. S. (2009). Sexually transmitted diseases among adults who had been abused and neglected as children: A 30-year prospective study. American Journal of Public Health, 99 (Suppl. 1), S197-S203. 